\title{
Epilog: Warum eine Kulturgeschichte der Migration notwendig ist
}

\subsection{Eine neue Kultur der Betroffenheit}

Das, was ein Individuum allein betreffe, dürfe nicht bestimmender Teil öffentlicher Anliegen sein, meint Jeff Weintraub in seinen Reflexionen zur Unterscheidung von »öffentlich« und »privat«. Das Individuelle sei letztlich zu partikular, um für die Gesellschaft stehen zu können. Doch die große Unterscheidung zwischen »öffentlich « und "privat« ist mehrdeutig und vielseitig. Wenn beispielsweise mehrere Bürger zusammenkommen und sich austauschen, wird aus einer privaten Sphäre eine öffentliche. ${ }^{1}$ Aus diesem Grunde ist - so Weintraub - das öffentliche Leben in modernen Gesellschaften und die Unterscheidung von »öffentlich«/»privat" dafür da, durch Kommunikation und Artikulation von Interessen emotionale Überforderungen aus dem Privaten im öffentlichen Leben (»public life«) auszugleichen und ihnen Formen von Kollektivität (gemeinsame Interessen- und Meinungsbildungen) zu geben, Teil einer Gesellschaft zu sein. Die Grundlage dieses zivilgesellschaftlichen Prozesses beruht - so Weintraub weiter - auf der "sociability«, der Geselligkeit, der Soziabilität ihrer Mitglieder. So sind die drei zentralen Kriterien des öffentlichen Raums Sichtbarkeit, Kollektivität und eine beide verbindende Form der Soziabilität. Ersteres ist offen, aufgedeckt und zugänglich für alle und setzt sich ab von dem, was versteckt und heimlich ist. Das zweite berührt die Interessen eines Kollektivs von Individuen im Gegensatz zum Individuellen. ${ }^{2}$ Dennoch sind die Konstituenten des öffentlichen Lebens, Sichtbarkeit und Kollektivität, nicht ohne ihre Gegensätze, Heimlichkeit und individuelle Betroffenheit, zu denken, ja nicht existent. Es ist die Soziabilität, nach Georg Simmel die Geselligkeit, die beide Seiten zusammenführt. ${ }^{3}$

Der Zusammenhang von Sichtbarkeit und Kollektivität ergibt sich daraus, dass sowohl das, was in der Öffentlichkeit gezeigt und gesehen wird und als auch was nicht gezeigt und nicht gesehen wird, jeweils wandelbar ist. Mit dem Gezeigten und Gesehenen hängt zusammen, wer und was zusammengehört oder wer oder was sich zusammengehörig fühlt. Und wichtiger noch, wer mit wem in

\footnotetext{
1 Weintraub, Jeff (1997): »Theory and Politics of the Public/Private Distinction«. In: Public and Private in Thought and Practice. Perspectives on Grand Dichotomy, Chicago: University of Chicago Press, S. 1-42, hier S. 15.

2 Ebd., S. 5.

3 Siehe hierzu: Simmel, Georg (2019): Die Geselligkeit, In: ders.: Grundfragen der Soziologie. Individuum und Gesellschaft, Berliner Ausgabe, S. 50-70.
} 
Kontakt und in Austausch tritt. Im Zentrum dieses Prozesses der Veröffentlichung und Vergesellschaftung steht die Interaktion, die Begegnung mit dem Anderen. Daher nehmen kulturelle Marker als sichtbare Kennzeichen in diesem Prozess eine zentrale Funktion ein. Mit ihnen bestimmen die Formen ihrer Sichtbarmachung durch gemeinsam geteilte Räume, Übergangsbereiche, geschlossene Räume, Schwellen, offene Räume und Orte ebenfalls, was gesehen und nicht gesehen wird. Verändert man ihren Einsatz und Gebrauch, verändert sich auch die Unterscheidung zwischen »öffentlich « und »privat « in einer Einwanderungsgesellschaft und verändern sich letztlich auch die Formen ihrer Soziabilität. Wie ich zu Beginn dieser Kulturgeschichte festgehalten habe, folgt die Soziabilität dem Prinzip, dass »jeder [...] dem andern dasjenige Maximum an geselligen Werten (von Freude, Entlastung, Lebendigkeit) [gewährt], das mit dem Maximum der von ihm selbst empfangenen Werte vereinbar ist «. ${ }^{4}$ Damit aber dieses Verhältnis seine Form wahren kann, darf weder ein Beteiligter in übertriebenem Maße seine individuellen Interessen oder Problemlagen thematisieren, noch darf der Inhalt oder das Thema des Gesprächs, des Zusammenkommens zum ausschließlichen Zweck avancieren. ${ }^{5}$ Das heißt aber keineswegs, dass die Verhandlungen gesellschaftspolitischer Fragen und Inhalte fehlen müssen.

In der vorliegenden Kulturgeschichte bin ich davon ausgegangen, dass Literatur und Film auf der Verbindung von Sprechen und Öffentlichkeit und auf dem mehrschichtigen Verhältnis von »drinnen« und »draußen« basieren. Außerdem habe ich zu Beginn das Politische als Feld von der Politik als System unterschieden. Ich bin von dem Befund ausgegangen, dass die Bundesrepublik Deutschland eine Einwanderungsgesellschaft ist, deren Kulturgeschichte noch nicht geschrieben wurde, weil sie nie zum politischen System gehörte. Ihre Geschichte ist eine informelle und eine potenzielle, die ich über verschiedene Elemente - Orte, Kontakte, Sprechweisen, Handlungen, Auseinandersetzungen (Kämpfe) und Gestaltungen - in der und durch die Geschichte der Literatur, des Films, der Debatten und Theorien sicht- und lesbar gemacht habe. Aus einem mikrologischen Zugang, nämlich mit dem Blick auf Interaktionen, zeigte sich das Politische als ein aktives Feld, in dem Kommunikationen und Praktiken, aber auch ihre Störungen stattfanden und weiterhin stattfinden. Die Geschichte der Migration, der Begegnungen und der Interaktionen ihrer Akteure verweist in und durch ihren Wandel im Gegensatz zur aktuellen Konstellation - dem Aufkommen einer neuen Politik der Identität - auf eine dynamische Form, auf ein Feld der Möglichkeiten. Das eigentlich Politische liegt hier wie bei Weintraub,

4 Simmel (2019): S. 58.

5 Vgl. HÜtTERMANN (2018): S. 219. Vgl. auch: LANGENOHL (2015): S. 110. 
Rosanvallon, Massumi und Simmel in der Konzentration auf die dynamische Form. ${ }^{6}$ Darin steckt ein »mehr-als « das, was subjektiv erfahren wird. Es gibt ein Gefühl, von den Anfängen bis heute in einem »größeren Kontext eingebettet zu sein ${ }^{7}{ }^{7}$

Ich habe eine Geschichte aufgezeigt, deren Bedingungen kultureller Produktion sich immer wieder verschoben und in jeder Phase jeweils eine eigene Form der Sichtbarkeit und Kollektivität produziert haben. Die aktuelle Phase seit 2014/2015, die ich am Schluss des letzten Kapitels bereits angedeutet habe, fordert die politische Aktivität und die Soziabilität im Sinne Rosanvallons und Simmels auf eine besondere Art und Weise heraus, weil in ihr Dynamiken der Schließung, Unterbrechungen und Entkontextualisierungen dominieren. Ähnlich wie Weintraub zielen auch Rosanvallon und Massumi auf die Konstitution eines Kollektivs, das durch Kontakt im öffentlichen Raum entsteht und am Ende zu einer Form und Erzählung findet. Rosanvallon beschreibt die politische Aktivität als einen Prozess, »durch den eine menschliche Gruppierung, die als solche eine bloße >Bevölkerung` darstellt, allmählich das Aussehen einer wirklichen Gemeinschaft annimmt«. Dieser Vorgang resultiere aus einem stets »umkämpften Prozess der Erarbeitung impliziter oder expliziter Regeln des kollektiv Zugänglichen und Verfügbaren«; er gebe dem »Leben des Gemeinwesens seine Gestalt «. ${ }^{8}$ Der aktuell zu beobachtenden Politik der Identität, die sich aus den Affekten der Angst, Ekel und Wut speist, soll so eine Politik der Zusammengehörigkeit entgegengesetzt werden. Ihre Grundlage ist der Affekt der Freude, die das Potential der Interaktion bejaht. ${ }^{9}$ »Grundsätzlich ist das >Gute affektiv als das definiert, was ein Maximum an Potenzial und Verbindung in die Situation bringt.« Für Weintraub, Rosanvallon und Massumi liegt der ethische Wert einer Handlung »in dem Beitrag zur Situation, darin wie sie diese verändert, wie es die Sozialität öffnet «. ${ }^{10}$ Entscheidend ist in diesem Prozess und im Zusammenhang der vorliegenden Kulturgeschichte der Migration, auf welchen Affekten die informelle Geschichte der Migration beruht. Die folgenden Ausführungen zu aktuellen publizistischen, literarischen und filmischen Arbeiten mögen veranschaulichen, warum es für den weiteren politischen Umgang mit der Migration und ihren Folgen in der Bundesrepublik notwendig und entscheidend ist, die Geschichte der Migration aufzuschreiben.

6 Siehe hierzu: Massumi (2010): S. 18.

7 Ebd., S. 30.

8 Rosanvallon, Pierre (2011/2012): »Für eine Begriffs- und Problemgeschichte des Politischen. Antrittsvorlesung am Collège de France«. In: Mittelweg 36, S. 43-65, hier S. 46.

9 Massumi (2010): S. 19.

10 Ebd., S. 34f. 
In Literatur und Film von den 1960er Jahren bis in die späten 2000er Jahre stehen bestimmte Formen der Interaktion und zugleich auch ihr Wandel im Zentrum: entweder durch Kontakt am Arbeitsplatz, in einer Kneipe, im Anliegen, eine türkische Frau aus einem türkischen Dorf auf eine deutsche Straße zu schicken, später dort »unerhört« aufzutreten oder an bestimmten Orten zu zeigen, was und wie man lebt. Demgegenüber unterbricht Güngörs individuelle Alltagserfahrung im Bioladen mit ihrer auch für sie unerwarteten hochaffektiven Reaktion unbeabsichtigt diese in der Geschichte von innen nach außen und von außen nach innen führenden Prozesse: Obwohl sie weder angesprochen wurde noch zur adressierten Gruppe gehört, macht sie eine tief in sie greifende Erfahrung der Diskriminierung. ${ }^{11}$ Dabei deutet sie ihre individuelle Erfahrung (Wut) ohne eine Form der expliziten Auseinandersetzung und Verhandlung als eine kollektive Erfahrung. Wenn Affekte wirken, wird intuitiv Geschichte aktualisiert, ohne dabei das Subjekt, die Umstände, die Situationen oder die Perspektiven anderer Beteiligter zu berücksichtigen. Es ist auch die Plötzlichkeit und Körperlichkeit des Affekts, die ihn von den Emotionen unterscheidet, die vielmehr von der Einschätzung, von Rahmungen der eigenen Position und gegenwärtiger Situationen bestimmt sind. Aufgrund dieser Filter und Rahmungen sind Emotionen zugleich der »unvollständige Ausdruck« eines Affekts. ${ }^{12}$

Die besondere Bedeutung des Affekts und der plötzlichen inneren Befindlichkeit, die aus der Geschichte der Migration kommt und ausbricht, finden wir, wie bereits angedeutet, nicht nur in den Texten von Güngör oder Akyün. Auch erdoğankritische türkeistämmige deutsche Journalisten wie Hakkı Tanrıverdi von der Süddeutschen Zeitung fühlten sich beispielsweise von Jan Böhmermanns Erdoğan-Gedicht als Türken verletzt und diskriminiert. ${ }^{13}$ Böhmermann mag zwar

11 Siehe hierzu insgesamt den Roman Ich bin Özlem von Dilek Güngör: GÜNGöR (2019).

12 Massumi (2010): S. 28.

13 Zwei Wochen vor Böhmermanns Sendung wurde in der Satiresendung Extra 3 der türkische Präsident mit dem Video Erdowie, Erdowo, Erdogan für seine repressive Politik kritisiert. Daraufhin bestellte die türkische Regierung zweimal den deutschen Botschafter in Ankara, Martin Erdmann, mit der Forderung ein, die deutsche Regierung solle das Video verbieten. In der deutschen Presse wurde dieses Vorgehen einhellig als äußerer Eingriff in die deutsche Pressefreiheit bewertet. Jan Böhmermann verlas daraufhin in seiner Sendung eine Schmähkritik an Erdoğan, um ihn darüber aufzuklären, was in Deutschland aufgrund des Artikels 5 des Grundgesetzes zur Kunstfreiheit Satire sei und was nicht. Eine Schmähkritik, die diffamierend und beleidigend sei, gehöre nicht dazu, was auch für sein vorgetragenes Gedicht gelte. Denn darin bezeichnet er den türkischen Präsidenten als "Ziegenficker« und als »Kinderschänder«. Böhmermann macht vor und während der Rezitation die Zuschauer und Erdoǧan darauf aufmerksam, dass das, was er jetzt vortrage, öffentlich nicht erlaubt sei. Anschließend erklärt Böhmermann Erdoğan, dass er gegen das Gedicht Klage einreichen könne, was tatsächlich auch 
zu Beginn der Sendung Neo Magazin Royale vom 31. März 2016 mit einem »Hülü« (turksprachige Verulkung von »Hallo«) die türkeistämmigen Deutschen angesprochen haben. Sein eigentlicher Adressat blieb jedoch Recep Tayyib Erdoğan. Tanrıverdi empfindet Böhmermanns Schmähkritik aber als »rassistisch«. Sie richte sich in Wirklichkeit nicht gegen Erdoğan, sondern gegen alle Türken. Schließlich verwende Böhmermann Begriffe wie »Ziegenficker«, mit dem man früher Türken in Deutschland diskriminierte. ${ }^{14}$

Auch der türkeistämmige deutsche Journalist Can Merey, der für die Deutsche Presse-Agentur (dpa) zwischen 2013 und 2018 Abteilungsleiter in Istanbul war, ${ }^{15}$ erhält über die Auseinandersetzung mit seinem Vater einen bestimmten Einblick in die Geschichte der Migration. Can Mereys Lebenslauf ist eigentlich das Abbild einer idealen Integration, wenn nicht sogar Assimilation: Die Mutter eine Deutsche, in Deutschland geboren, er studiert und ist mit einer deutschen Frau verheiratet. Anfang der 2000er Jahre war er als Leiter des Südasienbüros der dpa einer der ersten in dieser Branche mit türkischem Hintergrund. Doch die Gespräche mit seinem Vater für das Buch Der ewige Gast. Wie mein türkischer Vater versuchte, Deutscher zu werden in den Jahren 2017 und 2018 führten ihn am Ende seiner Recherchen ebenfalls dazu, sich als Teil eines unbestimmten Kollektivs, sich wieder als Türke zu begreifen. Ausgangspunkt von Der ewige Gast ist, dass Can nicht versteht, warum sein Vater Partei ergreift für Recep Tayyip Erdoğans Politik während und nach den Gezi-Protesten 2013 und zum Verfassungsreferendum in der Türkei und in Deutschland 2017. Anders als die Gastarbeiter kam sein Vater Ende der 1950er Jahre nicht zum Arbeiten, sondern

passiert. Am 17. Mai erließ die Pressekammer des Landgerichts Hamburg eine einstweilige Verfügung gegen Böhmermanns Gedicht, da es in Teilen beleidigend sei und deshalb Persönlichkeitsrechtsverletzungen vorlägen. 18 von 24 Zeilen des Gedichts dürfen demnach nicht mehr öffentlich von Böhmermann vorgetragen werden. Im August 2018 bestätigte das hanseatische Oberlandesgericht dieses Urteil mit dem Zusatz, dass es der Schmähkritik an »Schöpfungshöhe» fehle, um als Kunst begriffen werden zu können. Eine Revision ließ das Gericht nicht zu, da es sich beim Inhalt des Textes um »Beschimpfungen« handele. Eine Beschwerde könne aber beim Bundesgerichtshof eingereicht werden, was der Anwalt Böhmermanns auch tat. Siehe hierzu: Frankfurter Allgemeine Zeitung (2018): „Böhmermanns Erdoğan-Gedicht bleibt großteils verboten«. In: FRANKFURTER ALLGEMEINE ZEITUNG, 15.05.2018, https://www.faz.net/aktuell/ feuilleton/medien/jan-boehmermann-hamburger-oberlandesgericht-urteilt-ueber-schmaehgedicht-15591143.html (28.02.2018). Siehe auch: TAGESSCHAU (2019): "Streit um `Schmähkritikı. Böhmermann geht vor den BGH«. In: Tagesschau, 22.01.2019, https://www.tagesschau.de/kultur/ boehmermann-bgh-101.html (28.02.2018).

14 TANRIVERDI, Hakan (2016): »Böhmermanns Gedicht ist rassistisch. Seine Zeilen richten sich nicht gegen Erdoğan - sondern gegen alle Türken«. In: SÜDDEUTSCHE ZEITUNG. Jetzt, 02.04.2016, https://www.jetzt.de/fernsehen/was-boehmermanns-gedicht-ausloest (05.12.2018).

15 Seit dem 1. Juli 2018 leitet er das dpa-Büro in Washington, D.C. 
zum Studium der Betriebswirtschaftslehre nach Deutschland. Er lernte in den 1960er Jahren seine deutsche Frau kennen, Schweinebraten wurde seine Leibspeise, Bier sein Lieblingsgetränk, in den 1980er Jahren legte er die türkische Staatsbürgerschaft ab und nahm die deutsche an. ${ }^{16}$ Auch als Vertriebsleiter der schwäbischen Telekommunikationsfirma SEL identifizierte Tosun Merey sich nie mit den türkischen Gastarbeitern. Von Günter Wallraffs Buch Ganz unten (1985) fühlte er sich nicht angesprochen. Er hatte nicht einmal das Bedürfnis, für die diskriminierten und unterdrückten Türken Partei zu ergreifen, obwohl er ja selbst aus der Türkei stammte. Er begreift sich damals auch aufgrund seiner Bildung zugehörig zum internationalen Westen. Eine vergleichbare Selbstbestimmung und Positionierung in den 1980er Jahren konnte ich auch in der Literatur von Aysel Özakın aufzeigen. ${ }^{17}$ Die sozialstrukturelle Differenz trennt in beiden Fällen Welten und Kulturen voneinander. Doch diese Unterscheidung und die Umstände dieser Selbstpositionierung scheinen heute für Tosun Merey nicht mehr ausschlaggebend zu sein. Dank Erdoğan fühlt er sich nun als Türke angesprochen - und diskriminiert. ${ }^{18}$ Warum sich ein liberal denkender und lebender Mensch von Erdoğan vertreten fühlt, ist - so Can Merey - auf Diskriminierungen durch deutsche Behörden oder deutsche Arbeitskollegen zurückzuführen, die bis in die 1980er Jahre zurückreichen. Sein Vater sei trotz der diskriminierenden Erfahrungen in den 1980er Jahren Deutscher geworden und habe die türkische Staatsbürgerschaft dafür aufgegeben, weil beide Staaten damals befreundet gewesen seien. Die deutsche Staatsbürgerschaft anzunehmen und die türkische aufzugeben, »habe sich daher nicht wie eine Entscheidung gegen eine der beiden Seiten angefühlt«. Heute würde er es dagegen als einen »großen Verrat ansehen«, seinen türkischen Pass abzugeben. ${ }^{19}$

16 Siehe hierzu: Merey, Can (2018): Der ewige Gast. Wie mein türkischer Vater versuchte, Deutscher zu werden, München: Blessing, S. 41 u. S. 66. Siehe auch ebd., S. 40: „Während Gastarbeiter aus der Türkei unter Tage oder am Fließband schufteten, genoss Tosun den Luxus, tagsüber an der Universität Betriebswirtschaftslehre zu studieren und abends mit seinen Mitbewohnern über Gott und die Welt zu debattieren «.

17 Siehe hierzu: ÖzAKIN, Aysel (1983): Die Leidenschaft der Anderen, Hamburg: Luchterhand, S. 29. 18 Für die muslimisch-türkische Comunity und Parallelgesellschaft hält die Bloggerin Tuba Sarıca in ihrer Streitschrift Ihr Scheinheiligen fest, dass Erdoğan ihren Akteuren ermögliche, »ihren Hass gegenüber der westlichen Welt nachgeben « zu können. Siehe hierzu: SARICA (2018): S. 64. Es gehört zu Recep Tayyib Erdoğans politischer Erzählung, dass er aus ehrlichen und einfachen türkischen Verhältnissen kommt und die "schwarzen Türken « vertritt. Siehe hierzu: AKyol, Ciğdem (2018): Erdoğan. Eine kritische Biografie, Freiburg i. Br.: Herder. Siehe auch: YAVUZ, Hüdaverdi (2016): Reis, Türkiye.

19 Merey (2018): S. 300. 
Die Gespräche der Mereys führen so weit, dass der Sohn Can damit beginnt, seine eigenen Diskriminierungserfahrungen aufzulisten, die er zuvor nicht dokumentiert hatte. Sie reichen von der Lektüreerfahrung von Günter Wallraffs Buch Ganz Unten in den 1980er Jahren bis zu Aussagen deutscher Parlamentarier. So attestierte eine deutsche Politikerin dem Journalisten Merey im Jahr 2015 nach einem gemeinsamen Aufenthalt in der türkischen Stadt Cizre, dass er sehr gut Deutsch spreche. ${ }^{20}$ Es geht also gar nicht allein um den Vater in diesem Buch, sondern auch um den Erzähler. Weder am Anfang noch am Ende steht, anders als in den Reflexionen der 2000er Jahre, eine Ankunft. Die Herkunft ist nicht mehr etwas, das im öffentlichen Raum gefunden, als Material eingesetzt und damit integrativ verhandelt werden kann. Vater und Sohn sind nun durch ihre Vorgeschichte sewige Gäste`, sie sind Betroffene, obwohl sie sozialstrukturell privilegierte und leitende Positionen innehatten und innehaben. ${ }^{21}$ Mereys Buch setzt nicht etwa mit einer Diskriminierungserfahrung des Vaters ein, sondern mit einer eigenen, obwohl der Untertitel des Buches (Wie mein Vater versuchte, Deutscher $z u$ werden) klar die Biografie des Vaters thematisiert.

Can Merey ist als Journalist 2010 in Afghanistan und arbeitet dort an einer Dokumentation über die deutsche Bundeswehr. ${ }^{22}$ Als er in Nordafghanistan durch das Camp der Bundeswehr geführt wird, beschreibt ihm ein deutscher Offizier, dass man hier mit den Taliban umgehe »wie mit den Kanaken früher in der Schule. [...] Immer einen auf die Mütze geben, dann lernen sie’s irgendwann «. ${ }^{23}$ Merey merkt an, dass der Offizier überhaupt nicht geahnt habe, dass er gerade einen »Kanaken « durch das Camp führte. ${ }^{24}$

In den 1980er Jahren reagierten Şinasi Dikmen oder Aysel Özakın auf die Vorurteile ihrer deutschen Gesprächspartner mit der Lektüre der Wochenzeitung Die Zeit oder mit der Betonung ihrer Bildung. Sie zeigten, dass Türken sehr wohl

20 Ebd., S. 135.

21 Eine vergleichbare neue Identifikation mit dem Türkischen und den Türken begegnet man auch bei dem bekannten Nebenklägeranwalt im NSU-Prozess Mehmet Daimagüler. Im Jahr 2015 schreibt er, dass er »nicht wütend sein will«. Aber er sei es. Siehe hierzu: BECKER, Lisa (2012): »Mehmet Daimagüler. Ein Traum von Integration «. In: FRANKFURTER ALLGEMEINE ZEITUNG, 07.05.2012, https://www.faz.net/aktuell/beruf-chance/mein-weg/mehmet-daimagueler-ein-traumvon-integration-11739004.html?printPagedArticle=true\#pageIndex_0 (27.03.2019). Siehe auch: DAIMAGÜLER, Mehmet (2015): »Ich klage an. Der NSU-Prozess und meine Wut«, 12.11.2015, https://www.zeit.de/2015/44/nsu-prozess-wut (27.03.2019).

22 Siehe hierzu: Merey, Can (2010): Die afghanische Misere. Warum der Westen am Hindukusch zu scheitern droht, Weinheim: Wiley-VCH.

23 MeREY (2018): S. 9.

24 Ebd. 
gebildet und ein Teil des Westens sein können. ${ }^{25}$ Merey reagiert anders. Er klärt den deutschen Offizier nicht darüber auf, dass aus einem >Kanaken « offensichtlich ein deutschsprachiger Journalist werden kann. Er reagiert aber auch nicht wie die Gastarbeiter in den 1980er Jahren, die sich dachten, sollen die Deutschen doch sagen, was sie wollen, nächstes Jahr sind wir sowieso wieder in der Heimat. Zuletzt reagiert Can Merey aber auch nicht wie der Detektiv Cetinkaya in Dörries Film HAPPY BIRTHDAY TÜRKE!, der auf die diskriminierenden Aussagen des deutschen Hausmeisters im Jahr 1991 kontert, dass »die Putze gefälligst ihren Job machen und nicht unnötig rumlabern « solle; oder wie Zaimoğlu in der NDR-Talkshow 3NACH9 vom 8. Mai 1998. Dort wollte Gaby Hauptmann wissen, ob sie Zaimoğlu denn als »Kanake, der ein Buch geschrieben hat «, vorstellen dürfe. Er entgegnet, dass er sich selbst zwar als >Kanaken` bezeichne, sie ihn aber auf keinen Fall so nennen dürfe. Denn er würde mindestens mit einem »schiefen Blick» darauf reagieren. ${ }^{26}$ Zaimoğlu löste damals ein reges Gespräch zwischen Norbert Blüm, Heide Simonis und Giovanni di Lorenzo zu den Unterschieden zwischen der ersten und zweiten Generation der Einwanderer aus. Merey reagiert hingegen wie Güngör im Bioladen. Statt mit dem Offizier ins Gespräch zu kommen und weiterzusprechen, dass er selbst ein ehemaliger Kanake sei, um so Soziabilität zu genereien, hält er dieses Erlebnis Jahre später in einem Buch fest, in dem er nicht nur seinen Vater, sondern auch sich selbst als einen Betroffenen und »ewigen Gast« beschreibt: »Erst beim Schreiben ist mir bewusst geworden, wie sehr auch mein Leben von Tosuns Herkunft und von meinen türkischen Wurzeln geprägt worden ist - jenen Wurzeln, die ich einst am liebsten verdrängt hätte «. ${ }^{27}$ An die Stelle der direkten Reaktion in der Situation rückt eine zwar verdrängte, aber nie vergessene Geschichte. Sowohl für Can als auch für Tosun sind schlechte Erfahrungen aus den 1980er Jahren bestimmend. ${ }^{28}$ Can ist 13 Jahre alt, als er das

25 Siehe hierzu: Dikmen, Şinasi (1986): „Wer ist ein Türke«. In: ders.: Der andere Türke, Berlin: Express-Edition, S. 7-12, S. 9. ÖZAKIN, Aysel (1983): Die Leidenschaft der Anderen, Hamburg: Luchterhand, S. 29.

26 Siehe hierzu: Youtube (2010): »Feridun Zaimoğlu vs. Heide Simonis (1)«. In: YouTube, https://www.youtube.com/watch?v=wrV7adgbcMc , ab Min. 1:22 (01.03.2019).

27 MEREY (2018): S. 12.

28 Tatsächlich taucht im vielbeachteten Sammelband Eure Heimat ist unser Albtraum von 2019 der Verweis auf Diskriminierungserfahrungen von Großeltern, Eltern, farbigen Menschen und Ausländern in den 1980er und in den 1990er Jahren in vielen Beiträgen auf. Beispielsweise bezieht sich die Mitherausgeberin Fatma Aydemir in ihrem Text zu Arbeit paradigmatisch auf die Gedichte von Semra Ertan, die sich am 24. Mai 1982 in Hamburg aus Protest gegen den zunehmenden Rassismus öffentlich verbrannt hat. $\mathrm{Zu}$ ihrer eigenen Positionsbestimmung und zum Thema ihres Beitrags hält sie fest: »Migration ist immer ein Versprechen auf ein besseres Leben, einen German Dream. Der German Dream meiner Großeltern war, etwas Geld zur Seite zu legen 
erste Mal auf der deutschen Schule in Kairo von seinen deutschen Mitschülern abwertend als »Kanake« bezeichnet wird. Ein Spiegel-Artikel aus den 1980er Jahren über einen deutschen Wirt, der Türken mit zweisprachig ausgehängten Schildern (»Bu lokala Türkler giremez. Türken dürfen dieses Lokal nicht betreten«) den Zutritt verweigert, verstärkt seine Angst vor der Stimmung in Deutschland. Eindrücklich ist auch die Beschreibung, wie den jungen Can Merey die Lektüre von Günter Wallraffs Ganz unten beeindruckte, er sich aber damals mit seinem Vater nicht darüber austauschen kann. Denn sein Vater war kein Gastarbeiter. Heute schreibt er zu diesen Erinnerungen:

Jede Demütigung, jeder Angriff gegen den fiktiven Ali [in Ganz unten, Ö.E.] erschien mir gegen Menschen wie meinen Vater, meinen Bruder und mich gerichtet. Ich erinnere mich noch gut daran, wie ich die letzten Seiten bei einer Familienreise ins oberägyptische Luxor las, das Buch zur Seite legte und danach wie vom Donner gerührt war. Den Mut, meine Sorgen mit meinen Eltern zu besprechen, brachte ich nicht auf. Stattdessen versuchte ich, meine türkische Herkunft zu verdrängen - und begann, mich ihrer zu schämen. ${ }^{29}$

Wurde die Herkunft in den 1980er Jahren topografisch in türkischen Wohnungen, Cafés, Kulturvereinen, Imbissbuden und Restaurants abseits des öffentlichen Raums verortet, oder in kulturellen Formaten wie der Interkulturellen Woche in Gesellschaft und Soziabilität übersetzt ${ }^{30}$, erfolgt nun eine komplexe Identifikation mit ihr. An die Stelle des persönlichen Gefühls der Scham tritt die Wut, an die Stelle der Wahrnehmung der Gesamtsituation tritt ein kollektiv

und damit in der Türkei ein Stück Land zu kaufen. Der German Dream meiner Eltern war, ihren Kindern ein Studium zu ermöglichen und ein großes deutsches Auto zu fahren. Und was ist meiner? Ganz einfach: Ich will den Deutschen ihre Arbeit wegnehmen. Ich will nicht die Jobs, die für mich vorgesehen sind, sondern die, die sie für sich reservieren wollen - mit der gleichen Bezahlung, den gleichen Konditionen und den gleichen Aufstiegschancen. Mein German Dream ist, dass wir uns alle endlich das nehmen können, was uns zusteht - und zwar ohne dass wir daran zugrunde gehen. Rest in Power, Semra Ertan«. Siehe hierzu: Aydemir, Fatma (2019): »Arbeit«. In: Eure Heimat ist unser Albtraum, hg. v. Fatma Aydemir und Hengameh Yaghoobifarah, Berlin: Ullstein, S. 27-37, S. 37. Siehe hierzu auch diese Essays: IpPolito, Enrico (2019): »Beleidigung«. In: Eure Heimat ist unser Albtraum, S. 82-100. SHEHADEH, Nadia (2019): »Gefährlich«. In: Eure Heimat ist unser Albtraum, S. 122-129. Yun, Vina (2019): »Essen«. In: Eure Heimat ist unser Albtraum, S. 140-149. Siehe zu Semra Ertan: ErTan, Semra (2020): Mein Name ist Ausländer. Benim Adım Yabancı, Münster: edition assemblage, S. 10.

29 Ebd., S. 153. DIE ZEIT-Journalistinnen Özlem Topçu, Alica Bota und Khue Pham halten in ihrem Buch Wir neuen Deutschen von 2012 ebenfalls fest, dass sie sich in den 1980er Jahren für ihre Herkunft schämten und sie im öffentlichen Raum verbargen. Siehe hierzu: BotA, Alice/ PHAM, Khue/Topçu, Özlem (2012): Wir neuen Deutschen. Wer wir sind, was wir wollen, Reinbeck: Rowohlt, S. 102.

30 Siehe hierzu: EzLI (2020). 
greifender Affekt, der ohne symbolische Repräsentation das Kollektiv »wir und die Anderen« schafft. Am Ende der Lektüre wissen wir nicht, ob der Der ewige Gast ein Buch über den Vater, über die erste Generation, oder eines über den Sohn ist. Die erste Hälfte der Biografie konzentriert sich auf den Vater, die zweite auf den Sohn. ${ }^{31}$ Am Ende bekräftigt Can Merey Mehmet Daimagülers Aussage, dass er nicht wütend sein möchte, aber wütend sei. ${ }^{32}$ Man hat also den Eindruck, dass es sich um ein generationenübergreifendes Buch über »Türken« handelt, deren bindende Kategorie ein Affektzustand ist, eine körperlich-motorische Reaktion als der »Niederschlag einer Reminiszenz«. Ihre Grundlage liegt in der Geschichte der Migration in der Bundesrepublik Deutschland und ihrer Folgen. Auch in dem aktuellen Essayband Das Fremde, das in jedem wohnt von Zafer Şenocak begegnen wir dieser Form der Kollektivbiografisierung über Diskriminierungserfahrungen aus den 1980er Jahren:

\begin{abstract}
In einer überregionalen deutschen Tageszeitung las ich Ende 1981 einen Leitartikel, der mich tief verletzte. In diesem verglich der Autor Polen, ein kommunistisches Land, in dem das Militär geputscht hatte, um die Gewerkschaftsbewegung niederzuringen, mit der Türkei, zwei Länder mit einem Militärregime. Es klang in diesem Artikel so, als wäre das im Falle der Türkei annehmbar, ja geradezu zu erwarten, schließlich sei die Türkei ja kein europäisches Land. Doch Polen sei das Herzstück Europas und die Ereignisse dort eine beispiellose Tragödie. ${ }^{33}$
\end{abstract}

Kurz zuvor hatte Şenocak ganz allgemein festgestellt: Man müsse begreifen, dass aktuelle Debatten um die Frage, ob der Islam zu Deutschland gehöre, einen Eingriff in die Kindheit von Menschen in Deutschland darstellten; letztlich in ihre »Erinnerungen « und »Gefühle«, »die vielen Menschen wichtig sind und einen nicht unbedeutenden Anteil an ihrem Selbstwertgefühl haben «. ${ }^{34}$ Sein persön-

31 Vergleichbar verwoben sind in Şenocaks In deinen Worten. Mutmaßungen über den Glauben meines Vaters das Verhältnis des Vaters zum Islam und zur Migration und das des Sohnes, des Autors. Siehe hierzu: ŞENoCAK, Zafer (2016): In deinen Worten. Mutmaßungen über den Glauben meines Vaters, Berlin: Babel.

32 Siehe hierzu: MEREY (2018): S. 275.

33 ŞENOCAK, Zafer (2018): Das Fremde, das in jedem wohnt. Wie Unterschiede unsere Gesellschaft zusammenhalten, Hamburg: Edition Körber, S. 43.

34 Ebd., S. 29. Tatsächlich ist einer der Ausgangspunkte des Essaybandes Eure Heimat ist unser Albtraum, die Aussage des deutschen Innenministers Horst Seehofer von 2018, dass der Islam nicht zu Deutschland gehöre. Seine zweite Aussage aber, dass die Muslime sehr wohl zu Deutschland gehören und die Tatsache, dass die Bundeskanzlerin Angela Merkel ihren Innenminister korrigierte und äußerte, dass mit den Muslimen auch der Islam zu Deutschland gehöre, findet in dem Band von Aydemir und Yaghoobifarah keinen Widerhall. Siehe hierzu: AYDEMIR/YAGHOoBIFARAH (2019): „Vorwort«. In: Eure Heimat ist unser Albtraum, hg. v. dies., Berlin: Ullstein, S. 9-12. 
liches oben zitiertes Beispiel nimmt daher eine exemplarische Funktion für allgemeine Erfahrungen ein. Herkunft spielt bei Şenocak ebenfalls eine Rolle, die sie bisher nicht hatte. Sie ist an die Stelle der Beschreibungen des Angekommenseins getreten, von denen Şenocaks Essays in Deutschsein (2010) noch ausgegangen waren. Demgegenüber schreibt Şenocak nun in Das Fremde, das in jedem wohnt explizit von seiner »doppelten Identität« als körperliche Erfahrung und thematisiert gleichermaßen sein Türkisch- und sein Deutschsein. »Manchmal denke ich, dass meine Zunge eine andere Form bekommt, spreche ich Türkisch. Diese andere Form lässt sich einfach nicht negieren. ${ }^{35}$ Er sei außerdem zugleich in Deutschland und in der Türkei zu Hause, denn »diese doppelte Heimat ist keineswegs weniger attraktiv, als dazwischen oder nirgendwo zu sein «. ${ }^{36}$ Eigentlich hatte er gedacht, dass die Frage der Herkunft in seinem Körper »keine Basis mehr findet«. Er hätte alle seine »Wurzeln [...] ausgerissen«. Heute fragt er sich, ob er sie nicht doch »heimlich « aufbewahrt habe: »Auf dem Dachboden meiner Gedanken? « ${ }^{37}$. Zugleich ist ihm die Türkei auch fremd geworden. Als er sie im Jahr 2018 besucht, kommt er sich »wie ein Westdeutscher [vor], der die DDR besuchte «. ${ }^{38}$ Im Unterschied zu seinen vorherigen literarischen und essayistischen Werken, besonders aus den 1990er Jahren, begreift sich Şenocak als Teil eines in der Geschichte verwurzelten Kollektivs.

Wie man mit Geschichte hier umgehen muss, um die eigenen vergangenen Diskriminierungserfahrungen zu bewältigen, d. h. die Affekte in Emotionen zu verwandeln, die durch Weitersprechen, Identifikation und Empathie teilbar werden, werde ich abschließend näher erläutern. Zuvor möchte ich jedoch kurz darauf hinweisen, welche besondere erzählerische wie inhaltliche Bedeutung und zentrale Funktion Herkunft und Betroffenheit in den fiktionalen filmischen und literarischen Erzählungen seit 2015 haben.

Siehe zu Angela Merkel: Frankfurter AlLGEmeine Zeitung (2018): »Merkel: Auch der Islam gehört zu Deutschland«. In: FRANKFURTER ALLGEMEINE ZEITUNG, 16.03.2018; https://www.faz.net/ aktuell/politik/inland/angela-merkel-widerspricht-horst-seehofer-aussage-zum-islam-15497171. html.

35 Ebd., S. 70.

36 Ebd., S. 107.

37 Ebd., S. 203.

38 Ebd., S. 150. Nach den Reaktionen der türkischen Regierung auf den Putschversuch von 2016 stellte der damalige deutsche Finanzminister Wolfgang Schäuble ebenfalls den Vergleich mit der DDR an, den Erdoğan brüskiert zurückwies. Siehe hierzu: SüDdeutsche ZeItung (2017): »Schäuble vergleicht Türkei mit der DDR«. In: SüDDEUTSCHE ZEITUNG, 21.07.2017, (https://www. sueddeutsche.de/politik/tuerkei-konflikt-schaeuble-vergleicht-tuerkei-mit-der-ddr-1.3597581) (zuletzt 02.03.2019). 


\subsection{Der Blick in die Geschichte}

Er hätte selbst ein Opfer des NSU werden können, ihn hätte es auch treffen können und genau deshalb habe er den Film Aus DEM NichTs gedreht, konstatiert Fatih Akın 2017.9 Ein Jahr später erhielt der Film einen Golden Globe. Fatih Akıns Lebenswelt ist mit derjenigen der türkeistämmigen NSU-Opfer eigentlich nicht zu vergleichen: auf der einen Seite ein weltbekannter Filmregisseur, auf der anderen Blumenhändler, Imbissbesitzer oder Änderungsschneider. ${ }^{40}$ Akıns Betroffenheit findet allerdings Einzug in die erzählerische Grundstruktur des Films, in dessen Mittelpunkt die Perspektive der Protagonistin Katja Şekerci und Affekte wie Trauer, Verzweiflung und Wut der Betroffenen stehen. Zugleich ist der öffentliche Raum zu einem umkämpften und mitunter gefährlichen Terrain geworden. Anders als noch in den 2000er Jahren geht es in der Öffentlichkeit nicht mehr um Ankunft und kulturelle Verhandlung. Am Anfang des Films steht die Zerstörung eines Ortes, genauer des Büros für Steuerberatung und Übersetzung, das Katjas Ehemann Nuri Şekerci betreibt. Bei einem Nagelbombenanschlag kommen Nuri und der gemeinsame achtjährige Sohn Rocco ums Leben. Dieser brutale von Rechtsradikalen begangene Mord leitet das erste von drei Film-Kapiteln ein. Es trägt den Titel »Familie«. Nachdem die Polizei zunächst die türkische Drogenmafia verdächtigte, fasst sie schließlich das deutsche Neonazi-Ehepaar Edda und André Möller. ${ }^{41}$ Diese Festnahme bringt Katja zurück ins Leben, die aufgrund ihrer großen Trauer einen Selbstmordversuch unternommen hatte. Im zweiten Teil des Films (»Gerechtigkeit«) will sie Vergeltung vor Gericht. Doch aufgrund von Restzweifeln werden die Angeklagten frei gelassen. Dazu führt unter anderem die Aussage eines rechtsradikalen griechischen Hotelbesitzers, der dem

39 TAgeszeitung (2017): » Rache ist nichts Ethnisches« « Fatih Akın zum Film AuS DEM NicHTS«. In: taz, 19.11.2017, http://www.taz.de/!5460666/ (zuletzt 02.01.2019).

40 CASPARI, Lisa/Sundermann, Tom/Tröger, Julius (2018): "Sie sind nicht vergessen. Zehn Menschen hat der NSU laut Anklage ermordert. Sie passten nicht ins Weltbild der rechtsextremen Täter. Das sind ihre Geschichten«. In: DIE ZEIT, 11.07.2018, https://www.zeit.de/gesellschaft/zeitgeschehen/2018-07/nsu-morde-opfer-prozess (27.03.2019).

41 Die anfängliche Verdächtigung der Türkenmafia war einer von vielen Gründen, warum die NSU-Morde im Herbst 2011 so spät aufgedeckt wurden. Der erste Mord am Blumenhändler Enver Şimşek war bereits am 9. September 2000 in Nürnberg begangen worden. Es folgten bis 2007 sieben weitere Morde an türkeistämmigen beruflich Selbstständigen sowie Morde am griechischstämmigen Schneider Theodoros Boulgarides und zuletzt an der deutschen Polizistin Michèle Kiesewetter in Heilbronn. Der Nagelbombenanschlag in Akıns Film verweist auf den Nagelbombenanschlag vom 9. Juni 2004 in der Kölner Keupstraße, der ebenfalls vom NSU begangen wurde. Die Keupstraße gilt als Zentrum des türkischen Geschäftslebens in Köln. Siehe hierzu: MAUS, Andreas (2016): Der Kuaför aus der Keupstraße, Köln/Hannover: COIN FILM GmbH. 
deutschen Täterpaar ein Alibi verschafft, indem er bezeugt, Edda und André Möller seien zur Tatzeit im Urlaub bei ihm in Griechenland gewesen. ${ }^{42}$ Im Spielfilm wird gezeigt, wie im Gästebuch des Hotels (dem vorgebrachten Beweismittel) die Namen der Tatverdächtigen nachträglich eingetragen wurden. Katja und ihr Anwalt Danilo Fava sehen das, aber nicht die Richter oder andere Anwesende im Gerichtssaal. Als der Anwalt der Klägerin, der wie ihr Mann einen Migrationshintergrund hat, in den Saal ruft, dass man doch an der Handschrift sehen könne, dass die Namen der Angeklagten nachträglich eingetragen worden seien, stößt dies nicht auf Zuspruch. In der Erzähldiktion des Films wird hier zwischen einer schweigenden Mehrheit und einer betroffenen Minderheit unterschieden. Doch im Unterschied zu Tevfik Başers Film 40QM DeUTSChLAND ist die Mehrheit hier sichtbar und Teil der Verhandlung. Im letzten Filmkapitel »Das Meer« rächt sich Katja Şekerci, indem sie vor dem Wohnmobil der Möllers in Griechenland mittels selbstgebastelter Bombe einen Selbstmordanschlag begeht. Der Film beginnt und endet also mit einer Explosion, die jeweils Opfer fordert. ${ }^{43}$

Doch nicht nur die Zerstörung von Orten bzw. Geschäften steht im Gegensatz zu den Erzählungen der 2000er Jahre aus etwa SüPERSEKS, MEINE VERRÜCKTE TÜRKISCH HochZEIT, KEBAB CONNECTION, AUF DER ANDEREN SEITE oder Leyla und Die Tochter des Schmieds. Dort haben Orte erzählerisch wie integrationspolitisch eine äußerst konstitutive Funktion eingenommen, indem sie den Auseinandersetzungen, Interaktionen und den Handlungen einen Rahmen gegeben haben. In AUS DEM NICHTS erleben wir hingegen gleich zu Beginn, dass der öffentliche Raum unbehaglich und gefährlich ist. Schon der Regen im ersten Kapitel des Films ist so stark, intensiv und laut, dass die Akteure oft Schutz suchen müssen. Er spiegelt zudem die unerträgliche Trauer der Protagonstin wider. Die Unsicherheit und Schutzlosigkeit im öffentlichen Raum dokumentiert auch die emotional positivste Sequenz in diesem Film. ${ }^{44}$ Sie spielt im Gefängnis noch vor der Einblendung des ersten Kapitels. Dort heiratet Katja Nuri, der eine Haftstrafe wegen

42 Siehe hierzu: AkIn, Fatih (2017): Aus dem Nichts, Spielfilm, Hamburg: Bombero International $\mathrm{GmbH}$.

43 Can Mereys oben behandelte Biografie zu seinem Vater schließt damit, dass Tosun sich aufgrund der zunehmenden Türkenfeindlichkeit in Deutschland große Sorgen um seine Enkelin mache. In einer E-Mail schreibt er Can, er hoffe, »dass der türkische Opa für Mia irgendwann [...] nur noch eine Fußnote sein wird«. MEREY (2018): S. 307.

44 Unter dem Titel Wir Ungeschützten sind am 21.02.2020 in der Süddeutschen Zeitung Statements von 15 Kulturschaffenden zum Anschlag in Hanau veröffentlicht worden, darunter von Aras Ören, Shermin Langhoff, Said, Nuran David Calis und Burhan Qurbani, die von Angst, Wut und neuen Fremdheitserfahrungen bestimmt sind. Siehe hierzu: SÜDDEUTSCHE ZEITUNG (2020): »Wir Ungeschützten«. In: SÜDDEUTSCHE ZEITUNG, 21.02.2020; https://www.sueddeutsche.de/ kultur/anschlaege-hanau-kuenstler-intellektuelle-gastbeitrag-1.4809164. 
Drogenhandels absitzt. Er wird im Gefängnis BWL studieren und nach seiner Entlassung das erwähnte Büro für Steuerberatung und Übersetzung eröffnen. Mit einer Handkamera aufgenommen, wirkt die Heirat unmittelbar, und die Sequenz verdeutlicht den eigentlichen Marker und Träger der Verhandlung von Migration und Integration in Literatur, Film aber auch in theoretischen Arbeiten: Gefühle und Affekte. Wenn Heirat, Studium im Gefängnis, Freiheit und ein Geschäft in einem zentralen und regen Hamburger Stadtteil Interaktionen fördern und Sozialität öffnen, verkehrt die Ermordung des Ehemannes und das Ausbleiben der Gerechtigkeit vor Gericht den Affektzustand von Freude über Trauer in Wut, jedoch ohne für den zuletzt genannten Zustand eine symbolische Repräsentation zu haben. ${ }^{45}$

Zugehörigkeiten über anfangs individuelle Betroffenheit (Angst), die in einen kollektiven Affekt (Freude) umschlägt, generiert auch Selim Özdoğans Roman Wieso Heimat, ich wohne zur Miete aus dem Jahr 2016. Im Prolog scheint »Puddingshop Lale« in Istanbul eine wichtige Rolle zu spielen, denn dort haben sich die Eltern des Protagonisten Mustafa Krishna, eine Deutsche und ein Türke, im Jahr 1989 kennengelernt. Nach ein paar Jahren in Istanbul ziehen sie gemeinsam nach Freiburg in den Schwarzwald, wo Mustafa aufwächst. ${ }^{46}$ Sieben Jahre später trennen sich die Eltern und Mustafas Vater kehrt zurück in die Türkei. Mit Anfang zwanzig reist Mustafa seiner deutschen Freundin zuliebe in die Türkei. Sie meint, dass ihm seine Identität fehle, er hingegen fühlt sich vollständig. Die individuelle Suche nach einer national gerahmten Identität wird von Beginn an konterkariert: Der Protagonist leidet an »Hymnosomnie«, d. h. er reagiert körperlich auf nationalkulturelle Marker. So schläft er beispielsweise immer ein, wenn eine Nationalhymne erklingt. ${ }^{47}$ Er ist alles andere als ein Türke: Türken sind »Füchse«, und bei Mustafa Krishna wissen die Istanbuler aufgrund seiner Unkenntnis des Türkischen und seiner naiven, aber zugleich bloßstellenden Fragen nicht, ob er eine »Kuh» oder ein »Fuchs« ist. ${ }^{48}$ Außerdem äußert Mustafa in Istanbul jedem Türken gegenüber, dass dieser wirklich gut Türkisch spreche.

So komisch und gelungen Özdoğan kulturelle Stereotypen verhandelt, so ernst wird der Roman, sobald es um Gefühle und Affekte geht - etwa im Zusammenhang mit den Gezi-Protesten. ${ }^{49}$ Mustafas Mitbewohnerin Esra bittet ihn dringlichst darum, niemandem zu erzählen, dass sie einen Film über die Gezi-Proteste mache. Als er nach dem Grund fragt, antwortet sie: »Wir haben alle Angst. Du hast gesehen,

45 Siehe hierzu: AkIN (2017).

46 ÖzDoĞAN, Selim (2016): Wieso Heimat, ich wohne zur Miete, Innsbruck: Haymon, S. 11.

47 Ebd., S. 53.

48 Ebd., S. 129.

49 ÖzdoĞAN, Selim (2016): Wieso Heimat, ich wohne zur Miete, Innsbruck: Haymon, S. 32. 
auf der Istiklal ist jeden Tag mindestens ein Wasserwerfer, auch wenn dort nichts los ist. [...] Das hier ist nicht Deutschland: hab Angst «. ${ }^{50}$ Obwohl Mustafa von seinen türkischen Mitbewohnern nicht als richtiger Türke akzeptiert wird, macht ihn seine emotionale Reaktion auf diesen Film zu einem der ihrigen.

Ich wusste nicht, dass die Polizei Tränengas in die Wasserwerfer gefüllt hat. Ich wusste nicht, dass sie Tränengasgranaten in das Deutsche Krankenhaus hineingeschossen haben. Ich wusste nicht, wie viele Verletzte in der Dolmabahce-Moschee behandelt wurden. Und wie schwer sie verletzt waren. Bei manchen Bildern habe ich weggeschaut und mir gewünscht, sie würden eine Hymne spielen. [...]

Was ich mir merken kann, ist, dass während der Gezi-Proteste in der Türkei mehr Tränengas eingesetzt wurde als sonst im ganzen Jahr weltweit.

Ich könnte weinen, als der Abspann läuft, ich könnte weinen, aber gleichzeitig habe ich das Gefühl, ich müsste aufstehen und etwas tun. Als würden die Bilder, die ich gesehen habe, irgendetwas in mir bewegen, verschieben, und ich muss dann raus und die Welt geraderücken. [...] Ich will rausgehen, sage ich. Ich will irgendetwas machen. Mich bewegen. Menschen umarmen, Gesichter sehen, die Arme ausbreiten, springen .... ${ }^{51}$

Mustafa Krishna identifiziert sich mit den Gezi-Protesten, während er zu seinem türkischen Vater, seinem deutschen Onkel oder seiner deutschen Freundin keine derartige Beziehung aufbauen kann, wie er sie zu seiner türkischen WG in Istanbul unterhält. Aufgrund des Mangels an symbolischer Repräsentation endet der Roman mit einem Epilog, der das Erzählte und Gezeigte nicht in eine Parabel zu übersetzen vermag. ${ }^{52}$ Wenn es in diesem urkomischen und zugleich tiefernsten Roman eine zentrale Botschaft gibt, dann würde sie lauten, dass weder das »Liken« von politischen Protesten oder ihr digitales Teilen für eine politische Teilhabe steht, sondern nur die tatsächliche Teilhabe im unsicheren öffentlichen Raum: Gezi »war ein Gedicht, geschrieben von Millionen Herzen«.53 Mustafa Krishna kann sich mit seinen Mitbewohnern in Istanbul und sie sich mit ihm identifizieren, weil sie vermeintlich das Gleiche empfinden. Vor dem Epilog endet die Handlung des Romans damit, dass Mustafa Krishna in einem Flugzeug auf dem Weg nach Deutschland sitzt. Er kommt in der Erzählung nicht mehr an, weil es darum auch gar nicht mehr geht. Denn Mustafa wird noch etwas Zeit brauchen, um »zu verstehen, dass die Frage nach der kulturellen Identität

50 Ebd., S. 62.

51 Ebd., S. 133-135.

52 Im Epilog hat der »Chor der Ungläubigen« einen Kater, wahrscheinlich wegen einer durchzechten Nacht, und schafft es daher nicht mehr, »eine orientalistische Parabel zu erzählen«. Ebd., S. 243.

53 Ebd., S. 135. 
eine Ausweichstrategie ist. Denn die eigentlichen Fragen lauten: Unter welchen Bedingungen lebst du dort, wo du lebst? Wie ist das Geld verteilt, wie die Chancen und wie wird das begründet? «. ${ }^{54}$

Feridun Zaimoǧlus Epos Siebentürmeviertel spielt ebenfalls in Istanbul, allerdings zwischen dem Ende der 1930er Jahre (ein Jahr nach Atatürks Tod) und dem Anfang der 1950er Jahre. Ein deutscher Vater und sein Sohn Wolf müssen das Dritte Reich im Jahr 1939 verlassen, weil ersterer als Lehrer sich mehrmals über den Bart des deutschen Führers lustig gemacht hat. Sie werden von der türkischen Familie eines ehemaligen Lehrer-Kollegen im Istanbuler Siebentürmeviertel (Yedikule) aufgenommen. Auch auf den 800 Seiten von Siebentürmeviertel taucht die Identitätsfrage immer wieder auf. Und wie in Wieso Heimat, ich wohne zur Miete geht es auch hier nicht um einen Identifikationsprozess, an dessen Ende ein klares Ziel steht. Wolf ist sich vielmehr - wie Mustafa Krishna - von Anfang an klar darüber, wohin er gehört. Im Prolog berichtet der Ich-Erzähler Wolf, dass dieses Siebentürmeviertel sein Land sei, allerdings ein Land, das alles andere als sicher sei. ${ }^{55}$ So beginnt der Roman auch schon mit einem Straßenkampf seiner türkischen, tscherkessischen und griechischen Freunde gegen »größere Brüder «; da ist der Protagonist gerade einmal fünf Jahre alt. Er endet mit der Ankündigung neuer Straßenkämpfe 16 Jahre später: Nach dem Tod seines »türkischen Vaters«, des größten Feindes im Viertel, kündigt der Sohn Wolf an, dass der Kampf noch lange nicht vorbei sei. ${ }^{56}$

Auch nach dem Ende des Krieges will Wolf in seinem Viertel bleiben. Obwohl ihn die Nachbarn, seine Straßenbrüder und die Händler des Viertels als jemanden bezeichnen, der drei Väter hat (Abdullah, den Deutschen und Hitler), kommt es an keiner Stelle im Roman dazu, dass Wolf seine emotionale Verbundenheit zum Viertel in Frage stellt. Allerdings ist Yedikule kein Ort der Ankunft oder der sicheren Heimat für Wolf, sondern vielmehr ein Territorium, auf dem er sich behaupten und das er sich erkämpfen muss. ${ }^{57}$ Zaimoğlus bislang umfangreichstes Buch

54 Ebd., S. 242.

55 Siehe hierzu: ZaimoĞLu, Feridun (2015): Siebentürmeviertel, Köln: Kiepenheuer \& Witsch, S. 10.

56 Siehe hierzu: Ebd., S. 794. Wolfs deutscher (biologischer) Vater musste zu Beginn des Romans die türkische Familie verlassen, weil Abdullah Bey eine Tochter im heranwachsenden Alter hatte und die Nachbarn im Viertel das Gerücht verbreiteten, sie sei das "Arierliebchen" (ebd., S. 22) des deutschen Mannes. Im Verlauf des Romans versucht er mehrmals, seinen Sohn nach Kriegsende wieder nach Deutschland mitzunehmen. Vgl. hierzu ZAIMoğLU (2015): S. 490 u. S. 593.

57 Siehe hierzu: Ebd., S. 793f. Einer vergleichbaren erzählerischen Struktur folgt der Film FAMILIYE. VON DER STRASSE FÜR DIE STRASSE von Kubilay Sarıkara und Sedat Kurtan, der allerdings auf der Lynarstraße in Berlin-Spandau spielt. Familien und Straßen stehen aber auch hier im 
ist also kein Bildungs- und Entwicklungsroman im klassischen Sinne, an dessen Ende der Protagonist oder der Erzähler irgendwo ankommt, sondern vielmehr die Darstellung einer postmigrantischen Gesellschaft in den 1940er und 1950er Jahren in Istanbul, bei der weder Herkunft noch Ankunft zentrale Rollen spielen. Im Mittelpunkt stehen stattdessen Fragen der Verteilung, des Besitzes und der Selbstbehauptung: »Juden, Armenier, Türken, Kurden, die Jungen sind alle hier geboren und aufgewachsen. Und doch kommt es mir so vor, als wären sie erst vor kurzem eingewandert«, stellt Wolf etwa fest..$^{58}$ Prozesse der Migration und ihre Folgen sind keine Fragen der Integration mehr, sondern Fragen der Identifikation - im Besonderen der Affekte, mit denen die Praxis der Identifikation nun verbunden ist.

Der Affekt und insbesondere die Diskriminierungserfahrung kennzeichnen auch Fatma Aydemirs Roman Ellbogen. Erneut trägt sich die Erzählung im Stadtteil einer Millionenmetropole zu: Berlin-Wedding. Wie in Aus DEM NicHTS oder Siebentürmeviertel ist das Verhältnis der Akteure zur Stadt geprägt von (versperrten) Zugängen, von Diskriminierungen und von Kämpfen auf unterschiedlichsten Ebenen; ganz anders als in den 1990er Jahren, als dieses Verhältnis noch ein organisches ${ }^{59}$ war oder in den 2000er Jahren, als die Akteure einer klaren Orientierung folgten. ${ }^{60}$ Die einzige deutschstämmige Bekannte der Ich-Erzählerin und Protagonistin Hazal ist Desiree, zu der erstere jedoch nie vordringen konnte: »Nie habe ich Desirees Wohnung von innen gesehen, aber oft habe ich mir vorgestellt, wie es drinnen aussehen könnte «. ${ }^{61}$ Dafür bekommen wir einen sehr genauen Einblick in Hazals Leben und in die Wohnung ihrer Familie: Hazals Vater fährt seit über zwanzig Jahren in Berlin Taxi, redet bei der Arbeit viel, während zu Hause dann sein »Laberakku leer [ist], weil alles schon gesagt ist «. ${ }^{62}$ Hazal selbst spricht kaum mit ihm. Mit 14 Jahren hasste sie ihn sogar, da er ihr damals ihre langen Haare abschnitt, weil sie unerlaubterweise bei ihrer alevitischen Freundin Gül übernachtet hatte. ${ }^{63}$ Der Hass scheint in der Erzählzeit des Romans vier Jahre später nachgelassen zu haben, doch ein Gespräch zwi-

Zentrum des Geschehens. Siehe hierzu: SARIKAYA, Kubilay/KIRTAN, Sedat (2018): Familiye. Von der Straße für die Straße, Europa Kino Company GmbH.

58 Siehe hierzu: Ebd., S. 461.

59 Siehe hierzu: ÖzDAMAR (1992), (1998); ZAimoĞLU (1995), (1998); ARSLAN (1997), (1998); AKIN (1998); YAVUZ (1998).

60 Siehe hierzu: KuRT (2003); ÖZdAMAR (2003); KARA (2004); AKyüN (2005); ZAIMoĞLU (2005); ALAKUŞ (2005); AKIN (2007); AKKUŞ (2008).

61 Aydemir, Fatma (2017): Ellbogen, München: Hanser, S. 8.

62 Ebd., S. 39.

63 Siehe hierzu: Ebd. 
schen beiden kommt dennoch nicht zustande. ${ }^{64}$ Wenn der Vater spricht, dann vom türkischen Präsidenten Erdoğan, mit dem er sich identifiziert. Hazal wäre es wichtiger, darüber zu reden, warum sie als fast Volljährige »immer noch Ausreden suchen muss, um abends wegzugehen $«{ }^{65}$ An ihrem 18. Geburtstag darf sie bei ihrer Freundin Elma übernachten. Mit weiteren Freundinnen - nur die kopftuchtragende Ebru kommt nicht mit - macht sich Hazal alkoholisiert auf den Weg zu einer Diskothek. Dort wird ihnen der Zutritt verweigert. Gül denkt betrunken darüber nach, wo sie jetzt noch hingehen, doch kommen sie und die anderen nicht darüber hinweg, dass ihnen der Einlass in die Diskothek verwehrt wurde. Gül sagt darauf zu ihren Freundinnen, »[w]ären wir aus Polen oder Spanien oder so, und hätten wir so dreckige Turnschuhe an, wären wir bestimmt reingekommen. Diese Bastarde!«, meint Hazals Freundin Gül. Vor diesem verweigerten Zutritt hatte sich keine der jungen Frauen als Deutsch-Türkin, Türkin oder Muslima gesehen. Dennoch fühlen sie

es, wir fühlen dasselbe. Es ist so da und es ist so heftig, dass man es fast anfassen kann. Wut. Meine ist so groß, dass sie nicht mehr in mich hineinpasst. Sie droht meine Haut zu sprengen, mich von innen aufzuessen und wieder auszuspucken und wieder aufzuessen. Meine Wut berührt Elmas Wut, kocht und wuchert gemeinsam mit ihr, lässt sich von ihr immer weiter anstacheln. ${ }^{66}$

Kurze Zeit später töten die Freundinnen einen deutschen Studenten, der sie mit einem »dummen Spruch « angemacht hat. Den letzten, wahrscheinlich tödlichen Stoß versetzt ihm Hazal. Nach dieser Nacht flieht Hazal, ohne ein Wort mit ihren Freundinnen zu wechseln, nach Istanbul zu ihrem Facebook-Freund Mehmet, der vor Jahren in die Türkei abgeschoben wurde und dort ein äußerst tristes Leben führt. ${ }^{67}$

Wie Aus DEM NichTs ist auch diese Erzählung in drei Abschnitte aufgeteilt. Der zweite Teil beginnt damit, wie Hazal in Istanbul in der Wohnung von Mehmet und dem kurdischstämmigen Halit aufwacht. ${ }^{68}$ Die WG ist politisch gegen Erdoğan eingestellt. Freunde von Halit werden inhaftiert, weil sie die türkische Regierung in einem offenen Brief aufgefordert haben, den Krieg gegen die Kurden in Cizre zu beenden, den sie nach den verlorenen Wahlen im Jahr 2015

64 Auch die Protagonistin Özlem in Güngörs Roman Ich bin Özlem, schreibt, dass sie und ihr Vater im Unterschied zu früher kaum noch miteinander reden. Siehe hierzu: GüNGöR (2019): S. 32.

65 AYdemir (2017): S. 32.

66 Ebd., S. 114.

67 Ebd., S. $148 f$.

68 Ebd., S. 131. 
begonnen hatte. ${ }^{69}$ Wie Mustafa Krishna aus Özdoğans Roman kennt Hazal die politische und kulturelle Situation in der Türkei kaum. Das zeigt sich vor allem in einem kurzen Gespräch mit Halit, das jegliche Form kultureller Kompetenz desavouiert und die angefangene Interaktion abrupt unterbricht. Hazal ist in Berlin geboren und hat mit Ausnahme von ein paar Sommerurlauben mit der Familie in der türkischen Provinz nur in Deutschland gelebt. ${ }^{70}$ Bei einem Küchengespräch gelingt es ihr nun, fehlerfrei drei Sätze auf Türkisch zu sprechen. Für sie gebe es keinen Unterschied zwischen Kurden und Türken, weil »wir alle aus der Türkei kommen«.

Halit sieht mich an und beginnt, laut loszulachen. Es ist kein freundliches Lachen, sondern irgendwie verbittert. Es klingt, als wäre etwas Schreckliches passiert, so schrecklich, dass man nur darüber hinwegkommt, wenn man sich kaputtlacht. Das Lachen geht durch seinen ganzen Körper, und es will nicht aufhören. Ich werde richtig starr. Ganz langsam geht mir durch den Kopf, dass ich noch besser werden muss im Wutabschütteln, denn jetzt gerade droht es, nicht zu funktionieren. ${ }^{71}$

Halit reagiert nicht, indem er Hazal etwa die Beziehung zwischen Kurden und Türken erläutert. Ebenso wenig werden wir Leser von der Erzählerin über das Verhältnis und den Konflikt zwischen Türken und Kurden in der Türkei aufgeklärt. In Aydemirs Roman sind Reaktionen immer von Affekten bestimmt, die anders als Emotionen nicht mit verhältnismäßigen Vergleichen zu anderen Situationen oder Personen einhergehen. Ihren Mord des deutschen Studenten vergleicht Hazal später mit der Tötung eines türkischen Mannes durch seine Frau. Die von ihrem Ehemann mehrfach missbrauchte Täterin Cilem Doğan verteidigte sich beim Gerichtsprozess mit den Worten: »[H]ep Kadınlar mı ölecek? Biraz da erkekler ölsün. Namusum icin öldürdüm« (»Sollen denn immer die Frauen sterben? Ein

69 Siehe hierzu: Ebd., S. 196. Gegen diesen Krieg sprach sich auch die sehr bekannt gewordene Initiative Akademiker für den Frieden vom 11. Januar 2016 aus. 1128 größtenteils türkische Akademiker unterschrieben eine Petition an die türkische Regierung gegen ihre »Vernichtungs- und Vertreibungspolitik« im Osten der Türkei. Die türkische Regierung verstand diese Petition als terroristische Propaganda und als Beleidigung des türkischen Staates. Sie ließ 27 Wissenschaftlerinnen und Wissenschaftler verhaften. Angeklagt wurden alle Unterzeichner. Die Verfahren laufen bis heute. Siehe hierzu: SüDDEUTSCHE ZEITUNG (2016): »Erdoğan lässt Akademiker wegen Protestbrief verhaften «. In: SÜDDEUTSCHE ZEITUNG, 15.01.2016, https://www.sueddeutsche.de/ politik/tuerkei-erdoan-laesst-akademiker-wegen-protestbrief-verhaften-1.2820435 (19.03.2019).

70 Siehe hierzu: Aydemir (2017): S. 27. Die Protagonistin in Güngörs Roman Ich bin Özlem stellt ebenfalls fest, das sie alles, was sie über die Türkei, ihre Menschen und ihre Kultur weiß, weiß sie von »ein paar Wochen Sommerferien oder weil es [ihr] jemand anderes erzählt hat«, es sei „Wissen aus zweiter Hand«. Siehe hierzu: GÜNGÖR (2019): S. 81.

71 Ebd., S. 154. 
wenig sollen auch die Männer sterben. Ich habe für meine Ehre getötet. «). ${ }^{72}$ Hazal habe wie Cilem Doğan töten müssen, um zu überleben, obwohl ihr der deutsche Student außer einem »blöden Spruch « nichts angetan hat. $^{73}$

Hazal bekommt Besuch von ihrer Tante Semra, einer studierten Sozialarbeiterin, von der sie erfährt, dass Gül und Elma in Untersuchungshaft sitzen. Semra will Hazal davon überzeugen, zurück nach Deutschland zu kommen. Da sie nicht vorbestraft sei und Reue zeige, habe sie in Deutschland noch eine »Perspektive«. Doch wo Semra ein Gewissen und ein Bedürfnis nach Aufstieg und Ankunft vermutet - schließlich wollte Hazal einmal Ärztin werden ${ }^{74}$ - stößt sie nur auf eine schon lange existierende Wut. Hazal bereut die Tötung des Studenten keineswegs:

Weil solche Typen herumrennen und meinen, die Welt gehört ihnen. Weil die sich aufführen, wie sie wollen, weil die nie um irgendetwas kämpfen mussten. [...] Ich war wütend in der Nacht und hatte Angst und habe eben zugeschlagen, will ich ihr sagen. Aber das war nicht nur wegen der Nacht oder wegen dem Studenten, ich war schon vorher wütend, die ganze Zeit. ${ }^{75}$

Hazal erkennt, dass die Tante mit ihren »billigen Kartoffel-Sprüchen « sie nicht verstehen kann. Durch ihr Studium der Sozialpädagogik hatte sie in der Familie einen »Scheißsonderstatus«. Nach Hazal »labert [ihre Tante] irgendwas davon, dass sie selbst es auch nicht leicht gehabt hat. Und dass meine Eltern in Deutschland auch immer nur am Kämpfen gewesen sind«. Und Hazals Kopf ist »halb am Explodieren«, als ihre Tante »tatsächlich» das Wort »Migrationshintergrund « benutzt. $^{76}$ Hazal begreift endlich, »wie die Sache läuft«. »Kein Schwanz interessiert sich für uns, sie sehen uns nur, wenn wir Scheiße bauen, dann sind sie plötzlich neugierig. Wenn wir einen Thorsten vor die U-Bahn schmeißen, wollen sie auf einmal wissen, wer wir sind. ${ }^{77}$ Die Welt sei »scheißungerecht«: »Doch das liegt nicht an mir. Und dass ich das weiß, wird mir vielleicht nicht helfen so im praktischen Leben. Aber es hilft meinem Herzen ${ }^{78}{ }^{78}$ Sie fühlt sich einsam wie ihre kopftuchtragende Freundin Ebru, obwohl diese als überzeugte Muslima ein ganz anderes Leben führt. Sie hatte ihren Job als Zahnarzthelferin verloren, weil sie auf die weltweite Solidaritäts-

72 Siehe hierzu: KARAÇALI, Fatih (2015): »Hep kadınlar mı ölecek biraz da erkekler ölsün«. In: Milliyet, 10.07.2015, http://www.milliyet.com.tr/-hep-kadinlar-mi-olecek-biraz-da-gundem-2085701/ (02.12.2018).

73 AYdemir (2017): S. 126.

74 Ebd., S. 231.

75 Ebd., S. $244 \mathrm{f}$.

76 Ebd., S. 247.

77 Ebd., S. 249.

78 Ebd., S. 250. 
bekundung mit Charlie Hebdo einen Tweet mit den Worten absetzte, dass »jeder das bekommt, was er verdient \#fuckcharliehebdo«. Ihr Chef, Dr. Klinger, hat das gesehen und ihr am nächsten Tag mit der Aussage, dass er sie nicht mehr länger ausbilden kann, gekündigt. »Seitdem sitzt Ebru zu Hause." Als ihr dann die in Deutschland vollintegrierte Tante Semra gegenüber sitzt, versteht Hazal, was Ebru damit meinte, dass man Einsamkeit nicht teilen könne:

Ich denke an sie und an Gül und an Elma, und vielleicht fühlen wir alle ja dasselbe, vielleicht bin ich doch nicht ganz allein, vielleicht sind wir irgendwie miteinander verbunden. Das ist auf jeden Fall eine schöne Idee, ein beruhigender Gedanke, dass da jemand ist, der dieselben Kämpfe wie ich kämpft, nur woanders. Und ich, ich sitze ja auch in einer Zelle wie Gül und Elma, nur dass meine viel größer ist. ${ }^{79}$

Während die Gezi-Proteste in Selim Özdoğans Roman Wieso Heimat, ich wohne hier zur Miete die Freunde in der Türkei nicht in kultureller Hinsicht zusammenführen, sondern dies mittels Komik die Affekte Angst und Freude leisten, verstrickt sich Hazal immer mehr in ihre Welt aus Wut, Ressentiment und Einsamkeit. Sie kehrt nicht mit ihrer Tante nach Deutschland zurück, Halit wird inhaftiert und auch Mehmet lässt sie hinter sich, weil sie an seinem schlurfenden Gang abliest, dass er »nicht richtig auf den Boden« im öffentlichen Raum »tritt«: »So geht doch nur jemand, der leer ist. [...] Der nur sich selbst sieht, und sonst nichts « ${ }^{80}$ Im letzten Teil des Romans wohnt Hazal in einem Hotel und arbeitet in einem Café, um irgendwie über die Runden zu kommen. Der Roman endet mit dem Putschversuch in der Türkei am 15. Juli 2016. Von der Terrasse des Cafés in Üsküdar aus sieht sie, wie eine Gruppe des türkischen Militärs die BosporusBrücke, die Europa und Asien verbindet, besetzt. Doch sie sieht nicht wirklich das Militär, sondern ein »hellblaues Neon-H, gerahmt von Zickzack-Girlanden«, das für sie, für ihren Namen steht. Es steht für Hazalia, für das Land in ihr, das letzte Rückzugsgebiet. Als sie diesen Gedanken nachhängt, verlassen alle Gäste das Café und versuchen, nach Hause zu kommen. Der öffentliche Nahverkehr steht still; nur Militärfahrzeuge fahren durch die Stadt. Hazal versteckt sich draußen in einem Gebüsch. Dort entdeckt sie in ihrer Vorgeschichte eine Form der Geborgenheit, in Bildern und Szenen aus ihrer Vergangenheit:

Es fängt an, komisch zu riechen hier, ein bisschen wie Hazalia, wo das Messer liegt, mit dem mein Vater immer das Fleisch geschnitten hat, und meine langen schwarzen Locken, und auch der Studentenkörper und der rote See, aber wo keine Angst sein kann, weil es nämlich in Hazalia immer warm ist, als würde man ständig sich mit dem Rücken gegen die Heizung

79 Ebd., S. 251.

80 Ebd., S. 219. 
lehnen, oder als würde einen die ganze Welt umarmen, richtig umarmen, fest und ehrlich, nicht so mit zwei Zentimetern Sicherheitsabstand wie die Deutschen. [...] Das Gebüsch raschelt im Wind. Ich öffne die Augen, sehe ein Stück Nacht und lächle mir selbst zu. ${ }^{81}$

Versteckt im öffentlichen Raum vollzieht sich eine vermeintliche Öffnung aus dem Inneren des Körpers und aus den Erinnerungen Hazals heraus, eine ohne Gesellschaft und Soziabilität. ${ }^{82}$

Einer ähnlichen, jedoch anders gelagerten Verschiebung ins Innere begegnen wir in Şenocaks Essay Das Fremde, das in jedem wohnt. Wie Unterschiede unsere Gesellschaft zusammenhalten. Die Orte, an denen der Autor mit seinem Vater ins Gespräch kam, sind nach dessen Tod nicht mehr draußen. "Sie sind tief in mir«, schreibt Zafer Şenocak, dort, "wo das Gefühl der Verlassenheit in ein Gefühl der Geborgenheit übergeht«. Deshalb sitzt die Identität nicht mehr »auf der Haut«, sondern »wurzelt in der Tiefe, aus der Inspiration und Atmung entsteht«. Dort besteht ein »Gespinst aus kulturellen Bezügen«, die der Person »Halt und dem Leben Sinn geben «. Wie Hazal versteckt aus einem Gebüsch in die Nacht blickt, sieht auch Şenocak nur Dunkelheit. Die deutsche Einwanderungsgesellschaft kommt ihm vor wie ein »zugewachsener« und »dunkler Wald«. Dabei ist »der Boden des Waldes durchsichtig ${ }^{83}{ }^{83}$ Für die Augen unsichtbar führen im Boden »haarfeine Leitungen « von Wurzel zu Wurzel. »Sie verbinden dauerhaft Baum mit Baum. Wenn man in der Erde gräbt, sollte man das wissen.« Diese Perspektive verdeutlicht, dass selbst Şenocak das zuletzt so spannungsreiche Verhältnis zwischen türkischer und deutscher Politik als ein zusammengewachsenes versteht. Nur diejenigen Grenzen lassen sich überschreiten, die man zuvor anerkannt hat: »So ist das zwischen den Deutschen und Türken. Die mögliche gegenseitige Abneigung ist nur eine höhere Form der Anerkennung. Darin liegt Potential«. ${ }^{84}$ Dies betrifft auf der einen Seite Fragen der Zugehörigkeit und Zusammengehörigkeit. Auf der anderen Seite deutet das unsichtbare »Wurzelwerk« im Boden auch die Möglichkeit an, dass Venedig, Andalusien und Konstantinopel mit ihren Dichtern, Malern, Kaufleuten wiederentdeckt werden können. Der Blick nach unten in die Tiefe impliziert folglich keine Reise $»$ in eine exotische Welt, sondern eine

81 Ebd., S. 270f.

82 Nach ihren Diskriminierungserfahrungen im Supermarkt und nach dem Streit mit ihren Freundinnen und Freunden, der sie wie in einen »dicken Nebel [...] eingehüllt« hat, kommt es der Erzählerin Özlem in Dilek Güngörs Roman Ich bin Özlem so vor, dass sie zum ersten Mal ihre »eigene Stimme« wahrnimmt. »Ich hatte immer eine innere Stimme, aber ich hab ihr nicht getraut.« Siehe hierzu: GüNGÖR (2019): S. 123f.

83 Siehe hierzu: ŞENOCAK (2018): S. 90-94.

84 Ebd., S. 213f. 
$\mathrm{zu}$ unseren gemeinsamen Wurzeln in Europa ${ }^{85}$ Es wäre nach Şenocak mitunter diese historische Arbeit, die das neue kollektive Gefühl der Verlassenheit in eines der Geborgenheit übersetzen könnte. In diesem Zusammenhang gibt die Politikwissenschaftlerin Ciğdem Toprak in ihrer essayistisch und publizistisch ausgerichteten Studie Dies ist auch unser Land bemerkenswerte Reaktionen ihrer Interviewpartner auf den Anschlag 2020 in Hanau wieder.

Mich hat sehr berührt, was eine Hanauer Schülerin, selbst am Tag nach dem Anschlag, gesagt hat: > Die Mehrheit hat uns schon akzeptiert‘. Reibungen stellen unsere Zugehörigkeit nicht mehr infrage, sie wird auf beiden Seiten von Tag zu Tag mehr als Selbstverständlichkeit empfunden. `Es war für uns klar, dass wir einen Tag nach dem Anschlag von Hanau mit der Geschäftsführung und dem Betriebsrat zusammen eine Schweigeminute halten. Denn wir waren natürlich sehr betroffen`, erzählt der Arbeitsdirektor Ralph Wangemann von Opel. ${ }^{86}$

Toprak hat alle ihre Gesprächspartner, angefangen von Shishabarbesitzern über Sneakerdesigner, Mixed-Martial-Arts-Kämpfern, Integrationsbeauftragte bis hin zu Hip-Hop-Promotorinerinnen gefragt, ob sie nach den Anschlägen jetzt Angst hätten? »Nein. Alle haben darauf mit einem großen, selbstbewussten Nein geantwortet. Denn sie wissen: Das ist auch ihr Land. ${ }^{87}$ Als ein Fazit für Ihre Interviews für sich selbst hält sie am Ende ihrer Studie fest, dass ihr Heimatgefühl komplex ist; »aber eines ist sicher: Wir stehen heute selbstbewusster denn je zu Deutschland ${ }^{88} \mathrm{Zu}$ diesem optimistisch stimmenden Credo wirkt der ein Jahr zuvor erschienene Essayband Eure Heimat ist unser Albtraum wie ein Gegentext, deren identitätspolitische Grund- und Affektlage Diskriminierungen und Ressentiments sind. In ihm positioniert sich anstelle von Praktikerinnen und Praktikern eine Gruppe von Autorinnen, Journalistinnen und Kulturwissenschaftlern mit Einwanderungsgeschichte gegen eine deutsche und weiße "privilegierte Mehrheitsgesellschaft «, die einen Teil ihrer Mitglieder als »anders « markiert, sie nach Ansicht der Autorinnen und Autoren kaum schützt und nicht wertschätzt. ${ }^{89}$

Für Brian Massumi sind Affekte ebenfalls in der Lage, "Hoffnung « auszudrücken $;^{90}$ nach Freud artikulieren sie ein Erbe, eine Vorgeschichte. Die aktuelle integrationstheoretische Frage müsste lauten, welches Erbe und welche Affekte sich derzeit geltend machen. Der aktuelle Verlust des Außen, dieses

85 ŞENOCAK (2010): S. 175.

86 TOPRAK (2019): S. 243.

87 Ebd., S. 241.

88 Ebd., S. 240.

89 AYdEMIR/YaGHOOBIFARAH (2019).

90 MASSUMi (2010): S. 27. 
neue Aufkommen des Gefühls der Verlassenheit, den ich hier anhand einiger literarischer und filmischer Beispiele aus den vergangenen Jahren skizziert habe, erinnert an die 1980er Jahre, und er muss sich nicht unbedingt, wie bei Şenocak oder in Topraks Studie, in ein Gefühl der Geborgenheit oder der selbstbewussten komplexen Zusammengehörigkeit übersetzen. Dies verdeutlicht ein letzter Blick in Mereys Der ewige Gast und in Dilek Güngörs letztem Roman Ich bin Özlem. In Der ewige Gast artikuliert sich ein ähnliches Unbehagen in der deutschen Einwanderungsgesellschaft. Nachdem Can Mereys Eltern im Jahr 2013 ihr Hotel in der Türkei verkauft haben, leben sie abwechselnd in Bernau am Chiemsee und in Istanbul. Da er ein gutes Auskommen hat, in Bernau von den Nachbarn respektiert wird und deutsche Freunde hat, könnte Tosun »seine Sympathie mit wehenden Fahnen nach Deutschland tragen«. Das ist ihm aber nicht möglich, weil er »irgendwie« doch nicht als "gleichwertiger Mensch» behandelt wird. Dies hat nicht mit persönlichen Beleidigungen zu tun, sondern mit der allgemein vorherrschenden Meinung, die sich breit macht, wenn es um Türken geht. Diese verletze seine Würde und Tosun sagt, »echten Nazis sei er nur selten begegnet«. Doch sind nicht sie der »größte Feind «. Es sind die »rassistischen Gefühle, die unterdrückt werden, weil man weiß, dass es sich nicht schickt, rassistisch zu sein«. Tosun glaubt, »dass solche Gefühle bei einigen Deutschen vorhanden sind ${ }^{91}$

Gegen die politisch motivierte Trennung von deutsch und türkisch in den 1990er Jahren konnte man einfach vorgehen, indem man beispielsweise den diskriminierenden Gebrauch des Begriffs »Kanake« umkehrte, ihn positiv besetzte und damit markierte, dass man weder deutsch noch türkisch war. Tosun Merey ist wieder hundertprozentig Türke, obwohl er sich weder in der Türkei noch in Deutschland zu Hause fühlt; so wie Güngörs eigentlich durch und durch integrierte Protagonistin Özlem mit ihren Tränen zur Türkin wird, wenn der Begriff Ausländer an einer Supermarktkasse fällt oder in Gesprächen mit ihren deutschen Freunden der Begriff Brennpunktschule. In solchen Situationen tut sich ein Graben zwischen ihr und ihren Freunden auf, den es schon immer geben hat, so Özlem. " Ich bin mitten unter ihnen, aber bleibe die, die ich bin, die Andere «. ${ }^{92}$ In Mereys Familie sind die anhaltenden Verschlechterungen der deutsch-türkischen Beziehungen kein rein politisches und öffentliches Phänomen mehr, sondern spielen innerhalb der Familie eine Rolle: Je schlechter das Verhältnis zwischen der türkischen und deutschen Politik wird, »desto schlechter ist häufig auch die Stimmung beim Abendessen«. Konflikte auf politischer Ebene bergen

91 MEREY (2018): S. 289.

92 GÜNGÖR (2019): S. 110. 
»die Gefahr eines persönlichen Streits mit meinem Vater «, resümiert Can Merey. ${ }^{93}$ Sie gefährdet die Soziabilität auch nach innen. Als Fazit und Lösung für das akute Problem der Integration konstatiert er, dass die »Deutschtürken sich in erster Linie als Deutsche und erst danach als Türken fühlen« müssten. Das gelinge allerdings nur, wenn man ihnen das Gefühl vermitteln könne, »gleichwertige Mitglieder der Gesellschaft zu sein «. ${ }^{94}$ So sind die Fragen der Integration heutzutage nicht mehr einfach Fragen nach der Bildung, dem Zugang zum Arbeitsmarkt oder nach sozialen Kontakten, sondern mehr nach dem Gebrauch einzelner Worte, von Blicken, Begriffen, von Sprache, Kommunikation - letztlich von Affekten. ${ }^{95}$

Dass in den letzten genannten Werken Interaktionen unterbrochen werden, weil sich statt der bindenden Emotionen Neugier, Trauer oder Freude die Affekte Verzweiflung und Wut breit machen, nimmt den Betroffenen die Möglichkeiten $\mathrm{zu}$ verhandeln, d.h. über Auseinandersetzungen dem Unbehagen eine Form zu geben. Ein zentrales Problem ist dabei sicher, wie sich nun nach einer 60-jährigen Geschichte der Migration wechselseitiges Interesse und neue Neugier wecken lassen. Für eine festgefahrene politische und historische Situation sprechen einerseits die weiterhin im öffentlichen Raum zu hörenden Aussagen wie »Sie können aber gut deutsch!« oder »Wo kommen Sie denn ursprünglich her? «, andererseits aber auch die wütenden Reaktionen auf diese Aussagen, dass sie diskriminierend und rassistisch seien. ${ }^{96}$ Nur ein kontextbezogener Blick in die Geschichte der Migration zeigt, dass genau solche Aussagen und Fragen in den 1980er Jahren Dialoge und mitunter Freundschaften ermöglicht haben. Dialoge und Freundschaften waren die antidiskriminierende Reaktion auf den auslän-

93 Auch Tuba Sarıca konstatiert in ihrer Streitschrift, dass sie sich mit ihrer Mutter nur noch streite, weil sie wie die Parallelgesellschaft, die sie immer in Schutz nimmt, den Westen mittlerweile hasse. Dabei hat sie es früher geliebt, italienische Gnocchi, japanischen Basamtireis, Rotkohl und Salzkartoffeln für die Familie zu kochen. Heute allerdings sage sie, dass alles, was ihre Tochter schreibe, eine Lüge sei. »Das sagte meine Mutter mir in einem Kölner Restaurant, woraufhin ich ein Glas nahm, es auf dem Boden zerdepperte und ihr sagte, sie werde mich nie wiedersehen. Ich wusste einfach nicht, wohin mit der Enttäuschung darüber, dass selbst meine Mutter zu solch einer Unwahrheit fähig war, nur um die Parallelgesellschaft in Schutz zu nehmen. Aber Erdoğan war stärker.« Siehe hierzu: SARICA (2018): S. 98 u. 210.

94 Ebd., S. 305.

95 Ebd., S. 231.

96 Vu, Vanessa (2019): »Alltag Rassismus: Herkunft. Keine Antwort schuldig«. In: Zeit Campus, 27.02.2019, https://www.zeit.de/campus/2019-02/herkunft-identitaet-diskriminierung-rassismusselbstbestimmung (29.03.2019). KAZIM, Hasnain (2018): »\#MeTwo. `Wo kommst du eigentlich her ?. Viele Menschen mit dunklerer Hautfarbe beschweren sich, dass sie oft gefragt werden: ,Wo kommst du eigentlich richtig her? « Ist das Rassismus? Und darf man das fragen?«. In: DER SPIEGEL, 11.08.2018, http://www.spiegel.de/kultur/gesellschaft/metwo-wo-kommst-du-eigentlich-her-darf-man-das-fragen-a-1222620.html (29.03.2019). 
derfeindlichen Slogan »Türken raus«. Darauf basieren nicht nur die Filme DIE KÜMMELTÜRKIN GEHT (1985), ABDULlAH YAKUPOĞLU. WARUM HABE ICH MEINE TOCHTER GETÖTET (1986) oder YASEMIN (1987/88), sondern auch die Arbeit der Ausländerbeauftragten Barbara John in den 1980er Jahren in Berlin, die Geschichte der Interkulturellen Woche und das bekannte Kolloquium zur Standortbestimmung der Ausländerliteratur von $1984 .{ }^{97}$ Heute artikulieren die Aussagen »Sie können aber gut deutsch!« oder »Wo kommen Sie denn ursprünglich her?« ein neues kulturelles Unbehagen - Wut und Unsicherheit - auf beiden Seiten, das von Affekten, von »Niederschlägen einer Reminiszenz« bestimmt ist: Man nimmt sich aus der Geschichte nur das, was man braucht, berücksichtigt aber nicht die Folgen, ihre Grundlagen und die Geschichte ihrer Begegnungen. So verbleiben die Narrative der Migration, ihre eigentliche Ressource, eine Ressource der Soziabilität ungenutzt. Letztere ist es, die die zentralen Aspekte der Öffentlichtkeit, die Sichtbarkeit und Kollektivität mit ihren Gegenaspekten, dem Geheimnis und der betroffenen Individualität verbinden könnte.

Bessere Fragen wären sicherlich, wie man $\mathrm{zu}$ dem/der geworden ist, der/ die man heute ist - oder im Falle von Feridun Zaimoğlu, warum er Schriftsteller wurde. Mit seiner Antwort, dass er zu Hause viel geredet habe und draußen habe weiterreden wollen, habe ich die vorliegende Kulturgeschichte begonnen. Daraus hatte sich zunächst nicht geklärt, wo und wer draußen eigentlich zuhörte. Aber es gab in den 1990ern jedenfalls ein Draußen und damit Möglichkeiten der Begegnung, des »Plauderns « oder auch des »unerhörten« Auftretens. In ihrer Unbestimmtheit war Zaimoğlus Antwort offen für viele mögliche Formen der Interaktion und des Weitersprechens und des Aufbaus einer neuen sozialen Durchlässigkeit. Zentral war aber auch, dass man mit einem bestimmten Willen von irgendwo herkam, dort gesprochen hat und draußen weitersprechen wollte. Deshalb ist die Frage, wo man eigentlich herkommt, vielleicht sogar die beste, weil die Antwort darauf auch einfach lauten kann: von zu Hause. Und was dann alles zu diesem »zu Hause« gehört und was nicht, ob es darin behaglich oder unbehaglich ist, ob darin ein nicht eingeladener Fremder steht, oder ob das zu Hause eher die Schwelle zwischen drinnen und draußen ist, und man eigentlich gerne mehr Raum hätte oder ob man sich tatsächlich an den meisten Orten, die es in einem Land gibt, wohlfühlt, oder ob man zu Hause mit seinen Eltern nicht mehr sprechen kann, ist dann eine Frage der Erzählung und letztlich eine Frage der Soziabilität.

Ein zentrales Anliegen der vorliegenden Kulturgeschichte war es, mit der Darstellung der Transformation von Begegnung und Interaktion wieder Neugier

97 Siehe hierzu: AcKermanN/WeInREICH (1986); EzLI (2020). 
und Interesse auf die Geschichte der Migration zu wecken, um draußen wieder ins Gespräch zu kommen. Ob sich nun während oder am Ende dieser Gespräche eine Politik der Zusammengehörigkeit wird durchsetzen können oder vielleicht doch wieder eine Politik der Identität, wird sehr davon abhängen, wie diese Gespräche geführt werden und auf welchen Affekten sie beruhen. Die aktuellen Befindlichkeiten scheinen jedenfalls nicht einfach nur individuelle zu sein. Sie sind zum einen schon deshalb kollektiv, weil sie auf Affekten beruhen. ${ }^{98}$ Der Übergang von den individuellen Emotionen zu den kollektiven Affekten lässt sich zum einen sicher durch die zunehmende Verbreitung digitaler sozialer Medien verstehen, mit denen Gruppen entweder voneinander unterschieden, voneinander getrennt oder sich zu neuen Gruppen zusammensetzen. Zum anderen sind sie auf der deutschen wie auf der türkischen Seite nicht mehr von Ankunft und von Rückkehr bestimmt, lassen sich also nicht mehr einfach mit Integrationsprogrammen und Projekten oder mit symbolischen Repräsentationen in Form einer Erinnerungskultur verhandeln. Begriffe wie Transkulturalität, Postmigration und Hybridität werden hier auch nicht helfen. Die Befindlichkeiten reichen tief in die Geschichte der Migration zurück, die man kennen und ins Gespräch bringen muss, will man den aktuellen politischen Spaltungen begegnen und ihnen mit bindenden sozialen Kräften entgegentreten. Nicht zuletzt, um mit dem Wissen einer Kulturgeschichte der Migration den kollektiven Grad individueller Betroffenheiten erkennen, beschreiben und rahmen zu können.

98 In der klassischen Psychoanalyse wie auch in aktuellen philosophischen und medientheoretischen Reflexionen sind Affekte individuellen Emotionen vorgelagert. Sie werden als ein Wahrnehmen und Empfinden verstanden, deren Wirkkräfte ohne die Kategorie »eines intentional agierenden Subjekts« auskommen. Siehe hierzu: ANGERER, Marie-Luise (2017): Affektökologie. Intensive Milieus und zufällige Begegnungen, Lüneburg: meson press, S. 41. Siehe hierzu auch: GrEgG, Melissa/SeIGworTH, Gregory J. (2010): The Affect Theory Reader, London: Duke University Press, S. 3. ANGERER, Marie-Luise/Bösel, Bernd/OTt, Michaela (2014): Timing of Affect. Epistemologies, Aesthetics, Politics, Berlin: Diaphenes. 